\title{
UNIFORM TOEPLITZ MATRICES
}

\author{
I.J. MADDOX \\ Department of Pure Mathematics \\ Queen's University of Belfast \\ Belfast BT7 1NN \\ Northern Ireland \\ (Received October 19, 1988)
}

\begin{abstract}
We characterize all infinite matrices of bounded linear operators on a Banach space which preserve the limits of uniformly convergent sequences defined on an infinite set. Also, we give a Tauberian theorem for uniform summability by the Kuttner-Maddox matrix.
\end{abstract}

KEY WORDS AND PHRASES. Uniform Toeplitz matrix, strong slow oscillation, Tauberian theorems.

1980 AMS SUBJECT CLASSIFICATION CODES. 40C05, 40E05.

1. INTRODUCTION.

By $T$ we denote any infinite set of objects and we consider functions $f_{k}: T \rightarrow X$ for $k=1,2, \ldots$, where $(X,\|\|$.$) is a Banach space.$

The notation $f_{k} \Rightarrow f$ will be used to signify that $f_{k} \rightarrow f$ as $k \rightarrow \infty$, uniformly on $T$, which is to say that there exists $f: T \rightarrow X$ such that for all $\varepsilon>0$ there exists $k_{0}=k_{0}(\varepsilon)>0$ with

$$
\left\|f_{k}(t)-f(t)\right\|<\varepsilon \text {, for all } k>k_{0} \text { and all } t \in T \text {. }
$$

Now suppose that for $n, k=1,2, \ldots$ each $A_{n k} \in B(X)$, i.e. each $A_{n k}$ is a bounded linear operator on $X$. Then we shall say that $A=\left(A_{n k}\right)$ is a uniform Toeplitz matrix of operators if and only if:

$$
\sum_{k=1}^{\infty} A_{n k} f_{k}(t) \text { converges in the norm of } X
$$

for each $n \in N=\{1,2,3,4, \ldots\}$ and each $t \in T$ and

$$
\sum_{k=1}^{\infty} A_{n k} f_{k} \Rightarrow f
$$

whenever $\mathrm{f}_{\mathrm{k}} \Rightarrow \mathrm{f}$.

Following Robinson [1] and Lorentz and Macphail [2], if $\left(B_{k}\right)$ is a sequence in $B(X)$ we denote the group norm of $\left(B_{k}\right)$ by 


$$
\left\|\left(B_{k}\right)\right\|=\sup \left\|\sum_{k=1}^{p} B_{k} x_{k}\right\|
$$

where the supremum is over all $p \in N$ and all $x_{k}$ in the closed unit sphere of $x$.

By $C$ we shall denote the $(C, 1)$ matrix of arithmetic means, given by

$$
\begin{array}{cccccccc}
1 & 0 & 0 & 0 & 0 & . & . & . \\
\frac{1}{2} & \frac{1}{2} & 0 & 0 & 0 & . & . & . \\
\frac{1}{3} & \frac{1}{3} & \frac{1}{3} & 0 & 0 & . & . & . \\
. & . & . & . & . & . & . & .
\end{array}
$$

By $D$ we denote the Kuttner-Maddox matrix, used extensively in the theory of strong summability $[3,4,5]$ :

$$
\begin{array}{cccccccccccc}
1 & 0 & 0 & 0 & 0 & 0 & 0 & 0 & 0 & . & . & . \\
0 & \frac{1}{2} & \frac{1}{2} & 0 & 0 & 0 & 0 & 0 & 0 & . & . & . \\
0 & 0 & 0 & \frac{1}{4} & \frac{1}{4} & \frac{1}{4} & \frac{1}{4} & 0 & 0 & . & . & .
\end{array}
$$

In work on strong summability it is often advantageous to use the fact that, for non-negative $\left(p_{k}\right)$ the summability methods $C$ and $D$ are equivalent, in the sense that $p_{k} \rightarrow O(C)$ if and only if $p_{k} \rightarrow O(D)$.

In connection with Tauberian theorems we now introduce the idea of uniform strong slow oscillation.

Let $s_{k}: T \rightarrow X$ for each $k \in N$. Then we say that $\left(s_{k}\right)$ has uniform strong slow oscillation if and only if $s_{n}-s_{k} \Rightarrow 0$ whenever $k \rightarrow \infty$ and $n>k$ with $\mathrm{n} / \mathbf{k}=O(1)$.

In what follows we shall regard $s_{k}$ as the $k-t h$ partial sum of a given series of functions $\Sigma a_{k}=a_{1}+a_{2}+\ldots$, each $a_{k}: T \rightarrow x$.

2. UNIFORM TOEPLITZ MATRICES.

The following theorem characterizes the uniform Toeplitz matrices of operators which were defined in Section 1 .

THEOREM 1. $A=\left(A_{n k}\right)$ is a uniform Toeplitz matrix if and only if

$$
\begin{aligned}
& \sup _{n}\left\|\left(A_{n 1}, A_{n 2}, \ldots\right)\right\|<\infty, \\
& A \text { is column-finite, } \\
& \text { for each } n \in N, A_{n}:=\sum_{k=1}^{\infty} A_{n k} \text { converges, } \\
& A_{n}=I, \text { ultimately in } n \text {. }
\end{aligned}
$$

PROOF. We remark that in (2.3) the convergence is in the strong operator topology, and in (2.4), I is the identity operator on $\mathrm{X}$.

For the sufficiency, let $\mathrm{H}$ denote the value of the supremum in (2.1), let 
$\mathrm{n} \epsilon \mathrm{N}$ and $\mathrm{t} \in \mathrm{T}$. Then, for any $\varepsilon>0$ there exists $\mathrm{k}_{0}$ such that ||$f_{k}(t)-f(t)||<\varepsilon$ for all $k>k_{0}$.

Now for each $p \in N$,

$$
\sum_{k=1}^{p} A_{n k} f_{k}(t)=\sum_{k=1}^{p} A_{n k}\left(f_{k}(t)-f(t)\right)+\sum_{k=1}^{p} A_{n k} f(t),
$$

where we assume that $f_{k} \Rightarrow f$. By (2.3), as $p \rightarrow \infty$, we have

$$
\sum_{k=1}^{p} A_{n k} f(t) \rightarrow A_{n} f(t)
$$

Also, if $s \geq r>k_{o}$,

$$
\left\|\sum_{k=r}^{s} A_{n k}\left(f_{k}(t)-f(t)\right)\right\| \leq H \varepsilon,
$$

whence $\sum_{k=1}^{\infty} A_{n k} f_{k}(t)$ converges.

By (2.4) there exists $m \in N$ such that $A_{n}=I$ for all $n>m$, and by (2.2) there exists $\mathrm{n}_{\mathrm{o}}(\varepsilon) \in \mathrm{N}$ such that $\mathrm{A}_{\mathrm{nk}}=0$ for $1 \leq \mathrm{k} \leq \mathrm{k}_{0}$ and for $\mathrm{n}>\mathrm{n}_{\mathrm{o}}(\varepsilon)$.

Taking $\mathrm{n}>\mathrm{m}+\mathrm{n}_{\mathrm{o}}$ we have

$$
\sum_{k=1}^{\infty} A_{n k} f_{k}(t)=f(t)+\sum_{k=1+k_{0}}^{\infty} A_{n k}\left(f_{k}(t)-f(t)\right) .
$$

Since

$$
\left\|\sum_{k=1+k}^{\infty} A_{n k}\left(f_{k}(t)-f(t)\right)\right\| \leq \varepsilon\left\|\left(A_{n 1}, A_{n 2}, \ldots\right)\right\|,
$$

it follows by (2.1) that $\sum A_{n k} f_{k} \Rightarrow f$, which proves the sufficiency.

Now consider the necessity. Take any convergent sequence $\left(x_{k}\right)$ in $x$, with $x_{k} \rightarrow x$. Define $f_{k}(t)=x_{k}$ for all $k \in N$ and all $t \in T$, and define $f(t)=x$ for all $t \in T$. Then $f_{k} \Rightarrow f$ and so $\sum A_{n k} x_{k}$ converges for each $n$ and tends to $x$, whence the usual Toeplitz theorem for operators, see Robinson [1] or Maddox [6], yields (2.1) and (2.3) of our present theorem.

Next, suppose that $(2.4)$ is false. Then there exist natural numbers $n(1)<n(2)<\ldots$ with $A_{n(i)} \neq I$ for all $i \in N$. Hence there exist $x_{i} \in X$ with

$$
|| A_{n(i)} x_{i}-x_{i}||>0
$$

for all $i \in N$. Let us write $y(i)$ for the expression inside the norm bars in (2.5). Since $\mathrm{T}$ is an infinite set we may choose any countably infinite subset $\left\{t_{1}, t_{2}, t_{3}, \ldots\right\}$ of $T$. Then we define $f: T \rightarrow x$ by

$$
f\left(t_{i}\right)=x_{i} /\|y(i)\|
$$


for all $i \in N$, and $f(t)=0$ otherwise. If we define $f_{k}=f$ for all $k \in N$ then we certainly have $f_{k} \Rightarrow f$. But $A$ is not a uniform Toeplitz matrix, since for $\mathrm{n}=\mathrm{n}(\mathrm{i})$ we have by $(2.6)$,

$$
\left\|\sum_{k=1}^{\infty} A_{n k} f\left(t_{i}\right)-f\left(t_{i}\right)\right\|=\left\|A_{n} x_{i}-x_{i}\right\| /\|y(i)\|=1 .
$$

Hence, if $\mathrm{A}$ is a uniform Toeplitz matrix then (2.4) must hold, and a similar argument shows that (2.2) is necessary, which completes the proof of the theorem.

Since $C$, the $(C, 1)$ matrix, is not column-finite we immediately obtain:

COROLLARY 2. C is a Toeplitz matrix but not a uniform Toeplitz matrix.

However, since the elements of the Kuttner-Maddox matrix $D$ are non-negative and its row sums all equal 1 it is clear that the conditions of Theorem 1 hold, whence $D$ is a uniform Toeplitz matrix. Thus, whenever $f_{k} \Rightarrow f$ it follows that

$$
2^{-r} \sum_{r_{k}} \Rightarrow f,
$$

where the sum in (2.7) is over $2^{r} \leq k<2^{r+1}$ for $r=0,1,2, \ldots$. We also express (2.7) by writing $f_{k} \Rightarrow f(D)$.

The relation between $C$ and $D$ for uniform summability is given by:

THEOREM 3. $f_{k} \Rightarrow f(C)$ implies $f_{k} \Rightarrow f(D)$, but not conversely in general.

PROOF. Write

$$
c(n)=n^{-1} \sum_{k=1}^{n} f_{k}(t) \text { and } d(r)=2^{-r} \sum_{r} f_{k}(t) .
$$

Then we find that

$$
d(r)=\left(2-2^{-r}\right) c\left(2^{r+1}-1\right)-\left(1-2^{-r}\right) c\left(2^{r}-1\right),
$$

and it is clear that the right-hand side of (2.8) defines a uniform Toeplitz transformation between the $c$ and $d$ sequences.

For the last part of the theorem we may define real-valued functions on $\mathrm{T}$ by $f_{k}(t)=2^{r}$ when $k=2^{r}$ and $f_{k}(t)=-2^{r}$ when $k=1+2^{r}$, and $f_{k}(t)=0$ otherwise. Then $f_{k} \Rightarrow O(D)$. Now suppose, if possible, that $f_{k} \Rightarrow f(C)$, which implies $f_{k} \Rightarrow f(D)$. Hence $f=0$. But

$$
c\left(2^{r}\right)-\left(1-2^{-r}\right) c\left(2^{r}-1\right)=1 \text {, }
$$

contrary to the fact that $c(n) \rightarrow 0$.

3. A UNIFORM TAUBERIAN THEOREM.

By the remark following Corollary 2 we know that $f_{k} \Rightarrow f$ implies $f_{k} \Rightarrow f(D)$, but the example of Theorem 3 shows that the converse is generally false. The next result shows that uniform strong slow oscillation is a Tauberian condition for uniform $D$ summability. 
THEOREM 4. If $\left(s_{k}\right)$ has uniform strong slow oscillation and $s_{k} \Rightarrow f(D)$ then $s_{k} \Rightarrow f$.

PROOF. Without loss of generality we may suppose that $f=0$.

Take $n \in N$ and determine $r$ such that $2^{r} \leq n<2^{r+1}$. If $\varepsilon>0$ there exists $r_{0}$ such that if $2^{r} \leq k<2^{r+1}$ then

$$
|| s_{k}(t)-s_{n}(t)||<\varepsilon
$$

whenever $r>r_{0}$ and $t \in T$. Since

$$
2^{-r_{\Sigma}} s_{k}(t)=s_{n}(t)+2^{-r_{\Sigma}} r_{r}\left(s_{k}(t)-s_{n}(t)\right)
$$

we see that $s_{n} \Rightarrow 0$.

Our final result shows that the natural conditions $k_{k} \Rightarrow 0$ or $k a_{k} \Rightarrow 0(C, 1)$ are both Tauberian conditions for uniform $D$ summability, but that the restriction $k a_{k} \Rightarrow 0$ cannot be relaxed to the uniform boundedness of $\left(k a_{k}\right)$.

THEOREM 5. (i) If $k_{k} \Rightarrow 0$ or $k a_{k} \Rightarrow 0(C, 1)$ and $s_{k} \Rightarrow f(D)$ then $s_{k} \Rightarrow f$.

(ii) There exists a divergent series $\sum a_{k}$ with $\left(k a_{k}\right)$ uniformly bounded and $s_{k} \Rightarrow 0(D)$.

PROOF. (i) First note that $\mathrm{ka}_{\mathrm{k}} \Rightarrow 0$ does not generally imply $\mathrm{ka}_{\mathrm{k}} \Rightarrow 0(\mathrm{C}, 1)$ because $(C, 1)$ is not a uniform Toeplitz matrix by Corollary 2 . We shall show that $\mathrm{ka}_{k} \Rightarrow 0(\mathrm{C}, 1)$ is a Tauberian condition for $D$, the proof for $\mathrm{ka}_{k} \Rightarrow 0$ being similar. In fact we shall show that $k_{k} \Rightarrow 0(C, 1)$ implies that $\left(s_{k}\right)$ has uniform strong slow oscillation.

Let us write $a_{k}=a_{k}(t), s_{n}=s_{n}(t)$ and

$$
A_{n}=n^{-1} \sum_{k=1}^{n} k a_{k}
$$

with the assumption that $A_{n} \Rightarrow 0$. Then for $n>k \geq 1$, by partial summation,

$$
s_{n}-s_{k}=A_{n}-\frac{k}{n} A_{k}+\sum_{v=k+1}^{n-1}\left(v A_{v}-k A_{k}\right) / v(v+1) \text {, }
$$

whence

$$
\left\|s_{n}-s_{k}\right\| \leq \max \left\{\left\|A_{\nu}\right\|: k \leq v \leq n\right\}\left(1+\frac{k}{n}+2 \sum_{v=k+1}^{n} \frac{1}{v+1}\right) .
$$

If $\mathrm{n} / \mathrm{k}=O(1)$ then

$$
1+\frac{\mathrm{k}}{\mathrm{n}}+2 \sum_{v=\mathrm{k}+1}^{\mathrm{n}} \frac{1}{v+1}<2+2 \frac{\mathrm{n}}{\mathrm{k}}=O(1),
$$

and so $s_{n}-s_{k}=>0$, as required.

(ii) Define a numerical sequence $\left(s_{k}\right)$ by $s_{k}=0$ when $1 \leq k<4$, and for $n \geq 2$ 
define $s_{k}=0$ when $k=2^{n}$ and when $k=2^{n}+2 \times 2^{n-2} ; s_{k}=1$ when $k=2^{n}+2^{n-2}$ and $s_{k}=-1$ when $k=2^{n}+3 \times 2^{n-2}$. Otherwise define $s_{k}$ linearly, so that the graph of $\left(s_{k}\right)$ is a triangular-shaped wave. Then $\left(s_{k}\right)$ diverges and it is clear that $\sum_{\mathbf{r} \mathbf{s}_{k}}=0$ for all $\mathbf{r} \geq 0$. Also, it is easy to check that $k\left|a_{k}\right| \leq 8$ for all $k \geq 1$, whence our result follows on defining $s_{k}(t)=s_{k}$ for all $k \geq 1$ and all $t \in \mathrm{T}$.

\section{REFERENCES}

1. ROBINSON, A. On functional transformations and summability. Proc. London Math. Soc. $\underline{52}$ (1950) 132-160.

2. LORENTZ, G.G. and MACPHAIL, M.S. Unbounded operators and a theorem of A Robinson. Trans. Royal Soc. of Canada. XLVI (1952) 33-37.

3. KUTTNER, B. and MADDOX, I.J. On strong convergence factors. Quarterly J. of Math. (Oxford). 16 (1965) 165-182.

4. MADDOX, I.J. On Kuttner's theorem. J. London Math. Soc. 43 (1968) 285-290.

5. MADDOX, I.J. Inclusions between FK spaces and Kuttner's theorem. Math. Proc. Camb. Phil. Soc. 101 (1987) 523-527.

6. MADDOX, I.J. Infinite matrices of operators, Springer-Verlag, 1980. 


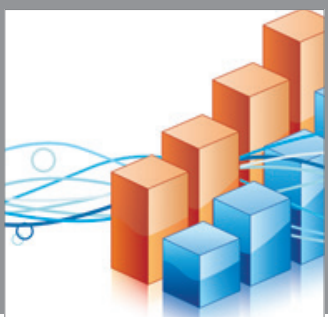

Advances in

Operations Research

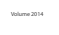

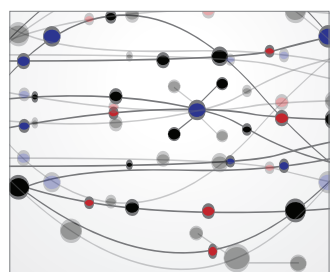

\section{The Scientific} World Journal
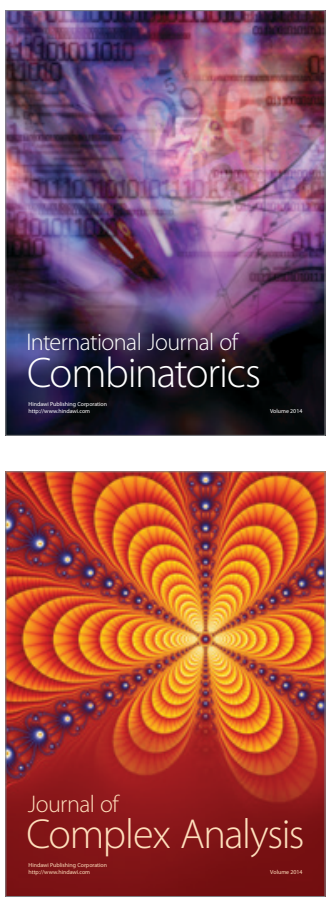

International Journal of

Mathematics and

Mathematical

Sciences
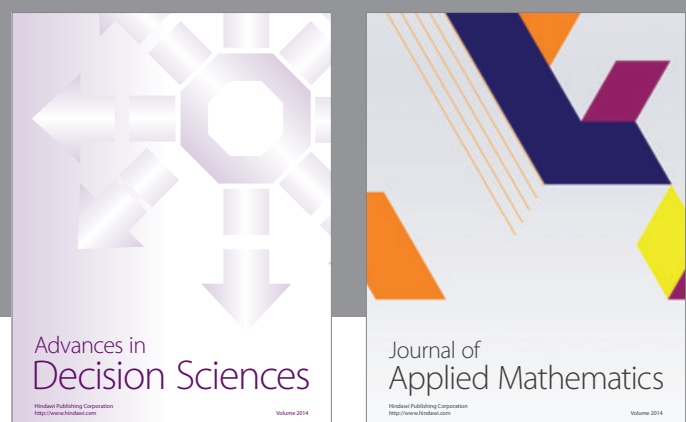

Journal of

Applied Mathematics
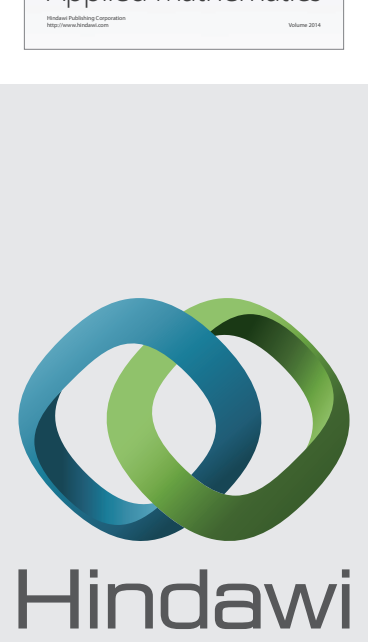

Submit your manuscripts at http://www.hindawi.com
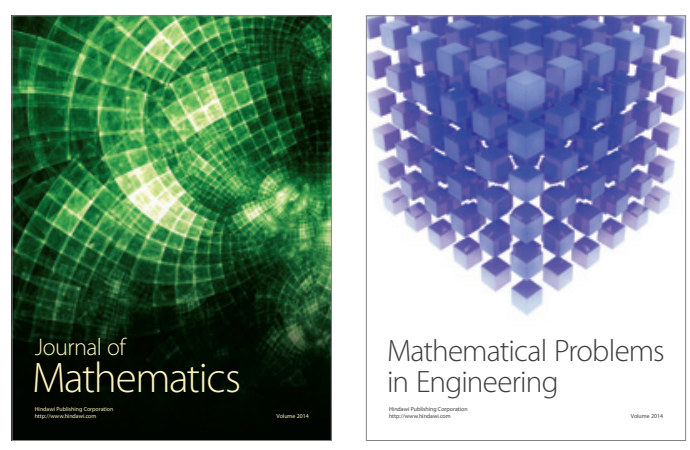

Mathematical Problems in Engineering
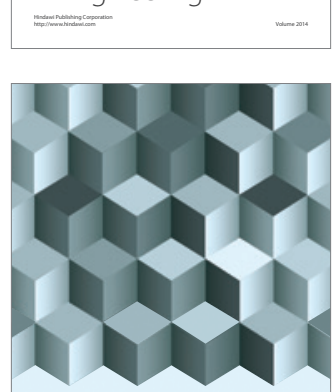

Journal of

Function Spaces
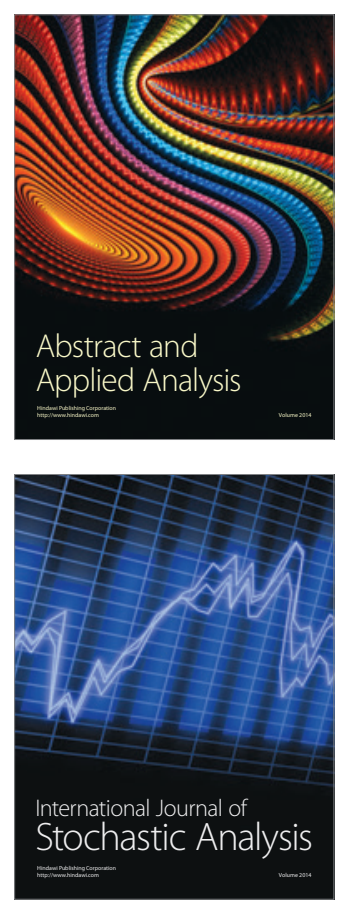

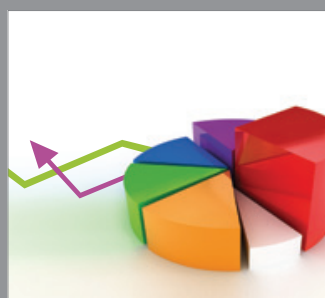

ournal of

Probability and Statistics

Promensencen
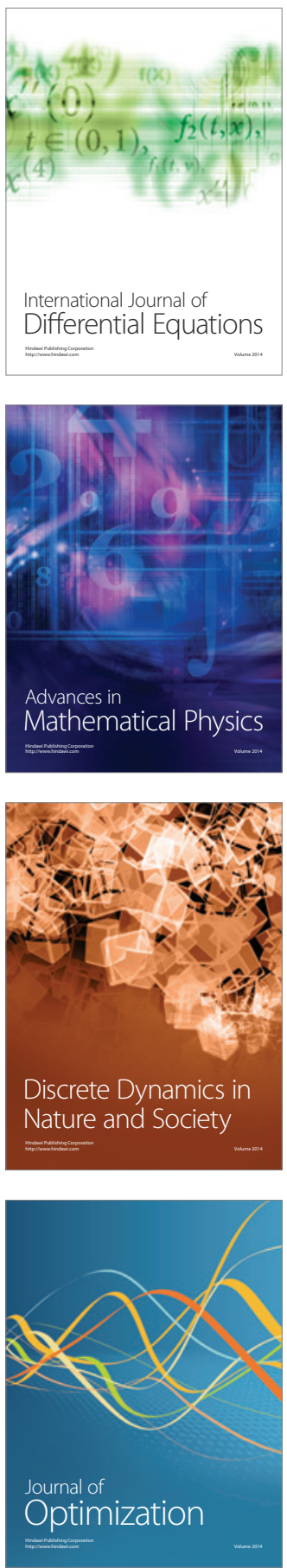